\title{
Correlative Imaging of Phase Separation in Fe2TiO4 Thin Films Prepared by Conventional Ga and Xe Plasma FIB Processing
}

Steven Spurgeon ${ }^{1}$, Bethany Matthews ${ }^{1}$, Ron Kelley ${ }^{2}$, Tiffany Kaspar ${ }^{1}$ and Scott Chambers ${ }^{1}$

${ }^{1}$ Pacific Northwest National Laboratory, Richland, Washington, United States, ${ }^{2}$ Thermo Fisher Scientific, Hillsboro, Oregon, United States

The development of new computing technologies depends on a detailed understanding of microstructure and its evolution in various processing conditions. Oxide materials in particular show considerable promise for applications such as spin injection and magnetic sensing, but their properties can be strongly degraded by common local fluctuations in structure and chemistry. Here we examine phase separation in ferrimagnetic $\mathrm{Fe}_{2} \mathrm{TiO}_{4}$ oxide thin films deposited on $\mathrm{MgO}$ (001) using molecular beam epitaxy. We quantify the sample morphology and microstructure using secondary electron (SE) imaging, as well as cross-sectional scanning transmission electron microscopy (STEM) and electron energy loss spectroscopy (EELS). We also evaluate the performance of conventional Ga-based focused ion beam (FIB) and Xe plasma FIB preparation methods, identifying differences resulting from each method. Our analyses unravel the complex microstructural evolution of this system and provide a window into structureprocessing relationships in an important class of functional materials.

Figure 1.A shows a representative SE image of the sample surface, exhibiting a rough, islanded morphology. Islands range in size from several 10s to $100 \mathrm{~s}$ of $\mathrm{nm}$ in a complex network. We compare this result to cross-sectional STEM high-angle annular dark field (STEM-HAADF) imaging, shown in Figure 1.B, which indicates that the islands rest atop a uniform layer of film growth. Initial film nucleation upon the $\mathrm{MgO}$ surface results in complete coverage, followed by increasing compositional fluctuations (indicated by the mottled contrast in the image) and eventual phase separation into a faceted morphology. We have conducted a comparison of the results of Ga-based and Xe plasma-based FIB sample preparation, as shown in Figures 1.B-C. Traditional Ga preparation results in significant surface accumulation of Ga, which is not present in the case of Xe plasma preparation. Such preparation artifacts must be mitigated to achieve the most accurate quantification of chemistry and composition. Figure 2 shows a detailed examination of the observed nanoscale phase separation using EELS. Large contrast changes are evident in the STEM-HAADF image (Figure 2.A), which result from almost complete chemical partitioning into Fe- and Ti-rich phases (Figures 2.B-D). Further high-resolution imaging confirms the presence of an unexpected $\mathrm{TiO}_{2}$ rutile phase, with implications for magnetic properties. Collectively our results demonstrate how a combination of state-of-the-art sample preparation and multi-modal imaging can generate deep insight into functional oxide systems. 

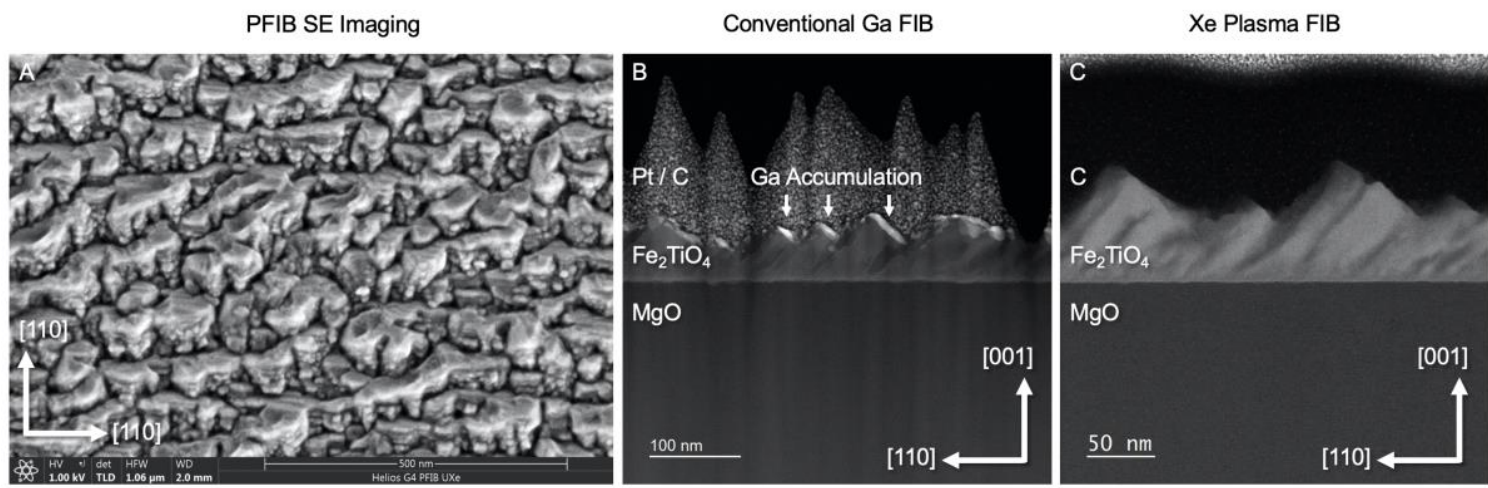

Figure 1. (A) SE image of the sample surface, exhibiting a complex two-dimensional island morphology. Cross-sectional STEM-HAADF images for conventional Ga (B) and Xe plasma (C) FIB prepared samples, illustrating surface Ga accumulation in the former and extensive nanoscale phase separation in both.
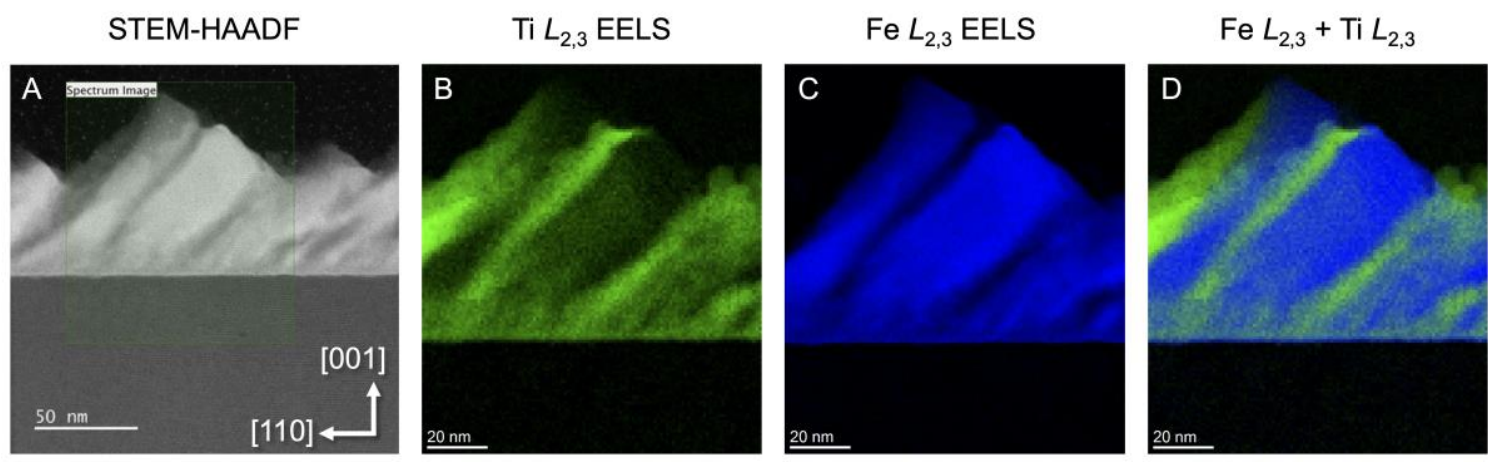

Figure 2. (A) Cross-sectional STEM-HAADF image of a phase separated region and (B-D) corresponding EELS maps for the Ti L2,3 edge, Fe L2,3 edge, and composite signals, respectively. 